\title{
Suppressing the secretion of exosomal miR-19b by gw4869 could regulate oxaliplatin sensitivity in colorectal cancer
}

\author{
Y. Y. GU, J. YU, J. F. ZHANG*, C. WANG* \\ School of Life Sciences, Nanjing University, Nanjing, China \\ *Correspondence: jfzhang@nju.edu.cn, chenwang@nju.edu.cn
}

Received March 6, 2018 / Accepted April 20, 2018

\begin{abstract}
Oxaliplatin is commonly used in managing malignancy, including colorectal cancer. While treatment often fails due to decreased drug sensitivity, the mechanisms involved are not clear. In this study, we investigate how exosomal miR-19b participates in oxaliplatin sensitivity and then prove that miR-19b down-regulates oxaliplatin sensitivity of sw480 cells. We found that suppressing the secretion of exosomal miR-19b with gw4869 promotes sw480 cell oxaliplatin sensitivity. Our combined results demonstrate for the first time that miR-19b regulates the oxaliplatin sensitivity of sw 480 cells and provides a unique mechanism mediated by gw4869 to modulate oxaliplatin sensitivity by suppressing exosomal miR-19b release.
\end{abstract}

Key words: exosome, oxaliplatin, colorectal cancer, miR-19b, gw4869

Colorectal cancer (CRC) has one of the highest incidences and mortality in China, and it also ranks second in 5-year survival rate [1]. Based on mathematical modelling, there will be a 124 percent rise in the incidence of colorectal cancer by 2030. Moreover, USA data shows increasing incidence of CRC in young people [2].

Oxaliplatin/irinotecan combined with 5-fluorouracil (5-Fu) is one of the current systemic treatments in CRC [3], and targeted therapies have been developed [4]. Despite technological advances, the prognosis of patients with metastatic CRC remains poor due to intrinsic or acquired tumor drug resistance [5]. Oxaliplatin is the first platinumbased anti-cancer drug approved for treating CRC. Although it causes cell death by controlling DNA synthesis, replication and transcription [6], resistance to oxaliplatin has become a major problem [7].

Exosomes are vesicles approximately $100 \mathrm{~nm}$ in diameter, and they can deliver their content, including protein, DNA and miRNA into the cytosol, thereby modifying the physiological state of the recipient cell [8]. Exosomes are important in cancer initiation and progression. It has been reported that exosomes can regulate the proliferation, invasiveness, EMT [9] and drug resistance of tumor cells and can promote angiogenesis, fibroblast activation, immune response [10], and pre-metastatic niche formation in distant sites [11].

MiRNAs were first identified in exosomes in 2007 [12], and they may function as a vehicle in intercellular commu- nication by transferring intercellular miRNAs $[13,14]$. By complementary base pairing with the 3 '-untranslated regions (3'-UTR), miRNAs can regulate post-transcriptional gene expression $[15,16]$.

Gw4869, the neutral sphingomyelinase inhibitor, is often used to block exosome generation [17], and it inhibits the release of exosomes from multi-vesicular bodies (MVBs) by inhibiting the MVB inward budding [18]. It was first used by Essandoh et al. to reduce inflammation and cardiac dysfunction [19], but the effect of gw 4869 on drug resistance and the molecular basis of this effect remain to be elucidated.

\section{Materials and methods}

Cells and reagents. The human colorectal carcinoma sw480 cell line was bought from Stem Cell Bank, Chinese Academy of Sciences. Cells were cultured in RPMI 1640 medium (Gibco) with $10 \%$ fetal bovine serum in a $37 \mathrm{C}$ incubator with $5 \% \mathrm{CO}_{2}$ supplementation. Oxaliplatin was purchased from Selleck, and gw4869 was purchased from Sigma.

Cell counting kit-8 assay (CCK-8) and EdU assay. After cells were seeded and kept overnight, different concentrations of oxaliplatin were added. Subsequently, $48 \mathrm{~h}$ later, the medium was changed to RPMI 1640 medium containing 10\% CCK-8 (Dojindo). Cells were then cultured in a $37^{\circ} \mathrm{C}$ incubator for $1 \mathrm{~h}$, and the optical density 
450 (OD450) was measured. The half-maximal inhibitory concentration (IC 50) values were calculated using GraphPad 6.0. The inhibition rate was calculated as follows: $\left[1-\left(\mathrm{OD}_{\text {test }}-\mathrm{OD}_{\text {blank }}\right) /\left(\mathrm{OD}_{\text {control }}-\mathrm{OD}_{\text {blank }}\right)\right] \times 100 \%$.

After cells were seeded and kept overnight, the culture medium was changed to contain $20 \mu \mathrm{M}$ oxaliplatin. An EdU assay kit (Ruibo) was applied to test the proliferation rate of the cells 48 hours later.

Isolation of exosomes. FBS contains significant quantities of vesicles that can influence cultured cell behavior [20]. To deplete these vesicles, FBS was subjected to $4 \mathrm{~h}$ of ultracentrifugation at $110,000 \mathrm{~g}$ (Beckman Coulter Avanti J-30I, USA), and the supernatant was filtered with a $0.22 \mu \mathrm{m}$ filter (Millipore) under aseptic conditions. Exosomes were collected from culture medium of treated or untreated cells. First, cells were seeded in equivalent amounts and cultured overnight and the medium was then changed to RPMI 1640 ( $2 \%$ exosome-free FBS) containing $20 \mu \mathrm{M}$ oxaliplatin; and not for controls. After $48 \mathrm{~h}$, the medium was centrifuged at $2,500 \mathrm{~g}$ for $20 \mathrm{mins}$ and at $10,000 \mathrm{~g}$ for $1 \mathrm{~h}$ to remove cells, debris and apoptotic bodies. The supernatant was subjected to 70 mins ultracentrifugation at $110,000 \mathrm{~g}$ at $4{ }^{\circ} \mathrm{C}$ after being filtered by $0.22 \mu \mathrm{m}$ filter to remove MV. Finally, PBS was used to re-suspend the pellet.

Identification and characterization of exosomes. Cells were lysed using RIPA buffer (Biochem) according to protocol. The protein concentrations of the cell extract and exosomes were assayed using the BCA protein assay kit (Thermo). The membranes with separated, and transferred protein was incubated with primary antibodies against Calnexin (Santa Cruz), TSG 101 (Abcam), CD63 (Abcam), CD9 (Abcam) and Actin (Abcam) at $4^{\circ} \mathrm{C}$ for $12 \mathrm{~h}$. HRP-conjugated secondary antibodies (Santa Cruz) were added to the bands and then detected using the ECL system.
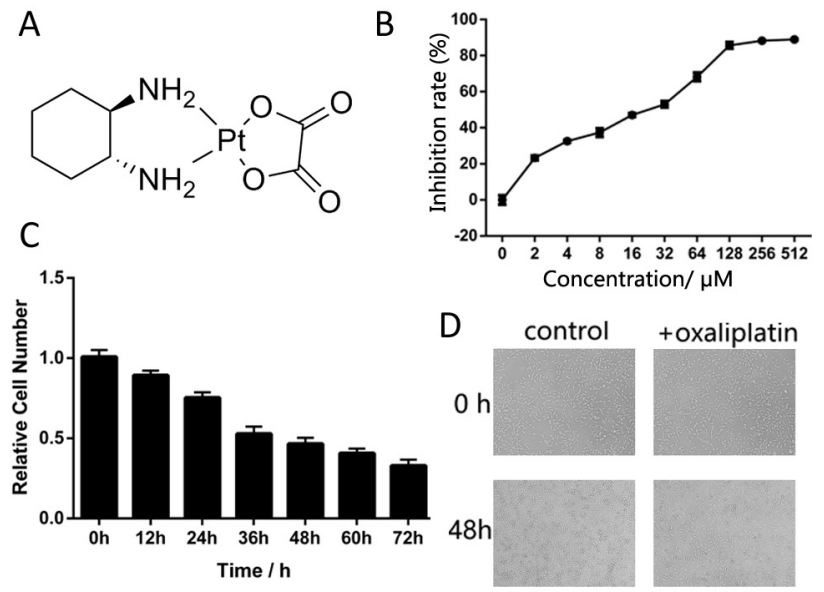

D
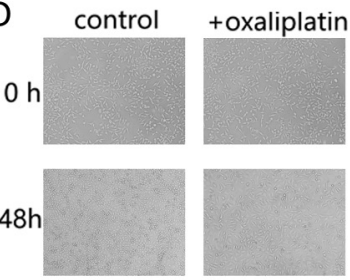

Figure 1. Effects of oxaliplatin on the proliferation of sw480 cells. A) The chemical structure of oxaliplatin. B) Oxaliplatin decreases cell proliferation in a dose-dependent manner. C) Oxaliplatin decreases cell proliferation in a time-dependent manner. D) Oxaliplatin does not change cell morphology.
The number and size of exosomes were directly tracked using the Nano platform (iZON Science). First, particles of known size were used to calibrate the NP100 nanopores of the measuring system and exosomes were then diluted with PBS to the proper concentration and added to the nanopores for measurement. Particle size and count measurements were performed using a particle analyzer and the Control Suite software.

miRNA overexpression and knockdown. To achieve miRNA over-expression and knockdown, miRNA mimics and inhibitors were used, respectively. Synthetic miRNA mimics and inhibitors were purchased from Ruibo. Cells were seeded in suitable plates and after reaching $80 \%$ confluency, Lipofectamine 3000 (Invitrogen) was used for transfection. Six hours later, the culture medium was changed from opti-MEM to RPMI-1640 containing 2\% FBS.

RNA isolation and QPCR. TRIzol (Invitrogen) isolated total RNA from cells and exosomes and QPCR was performed to detect the miRNA levels using TaqMan miRNA probes. After the reaction, we used fixed threshold settings to calculate cycle threshold $\left(\mathrm{C}_{\mathrm{T}}\right)$ data. The mean $\mathrm{C}_{\mathrm{T}}$ was calculated from the triplicate PCR results. U6 served an internal control in the cells and in the exosomes, synthetic peu-miR-2911 was spiked during exosomal RNA extraction for normalization. The ratio of miR-19b to U6/miR-2911 was determined by equation $2^{-\Delta \Delta \mathrm{CT}}$, which means that $\Delta \Delta \mathrm{C}_{\mathrm{T}}=\left(\mathrm{C}_{\mathrm{T} \text { miR-19b}}-\mathrm{C}_{\mathrm{T} \text { control }}\right)_{\text {sample }}-\left(\mathrm{C}_{\mathrm{T} \text { miR-19b}}-\mathrm{C}_{\mathrm{T} \text { control }}\right)_{\text {control }}$.

Statistical analysis. All western blotting and EdU assay images are representative of at least three independent experiments. QPCR and CCK-8 were performed in triplicate, and each experiment was repeated at least three times. The results are presented as the mean \pm SD and differences between groups were calculated by Student's t-test, with $\mathrm{p}<0.05$ statistically significant.

\section{Results}

Oxaliplatin inhibits proliferation of sw480 cells. Figure 1A shows the chemical structure of oxaliplatin. Different concentrations of oxaliplatin, ranging from $0 \mu \mathrm{M}$ to $100 \mu \mathrm{M}$, were used to treat sw 480 cells for $48 \mathrm{~h}$ (Figure 1B), and the results showed that the IC50 of oxaliplatin in sw480 cells was $20.14 \pm 1.04 \mu \mathrm{M}$. We used $20 \mu \mathrm{M}$ oxaliplatin to treat cells for $0 \mathrm{~h}, 12 \mathrm{~h}, 24 \mathrm{~h}, 36 \mathrm{~h}, 48 \mathrm{~h}$ and $60 \mathrm{~h}$. We found that oxaliplatin inhibited the proliferation of sw480 cells in a time-dependent manner (Figure 1C). Oxaliplatin changed both cell numbers and the morphology of sw480 cells (Figure 1D).

Exosome isolation and verification. To isolate the exosomes that cells secreted into the culture medium, we centrifuged as in Figure 2A. Western blot was used to evaluate the purity of exosomes. Figure $2 \mathrm{~B}$ showed that the exosomes secreted from sw480 cells present exosome markers, including TSG101, CD63 and CD9, whereas the exosome negative marker Calnexin was only identified in total cellular lysates; thus indicating the acceptable purity of 


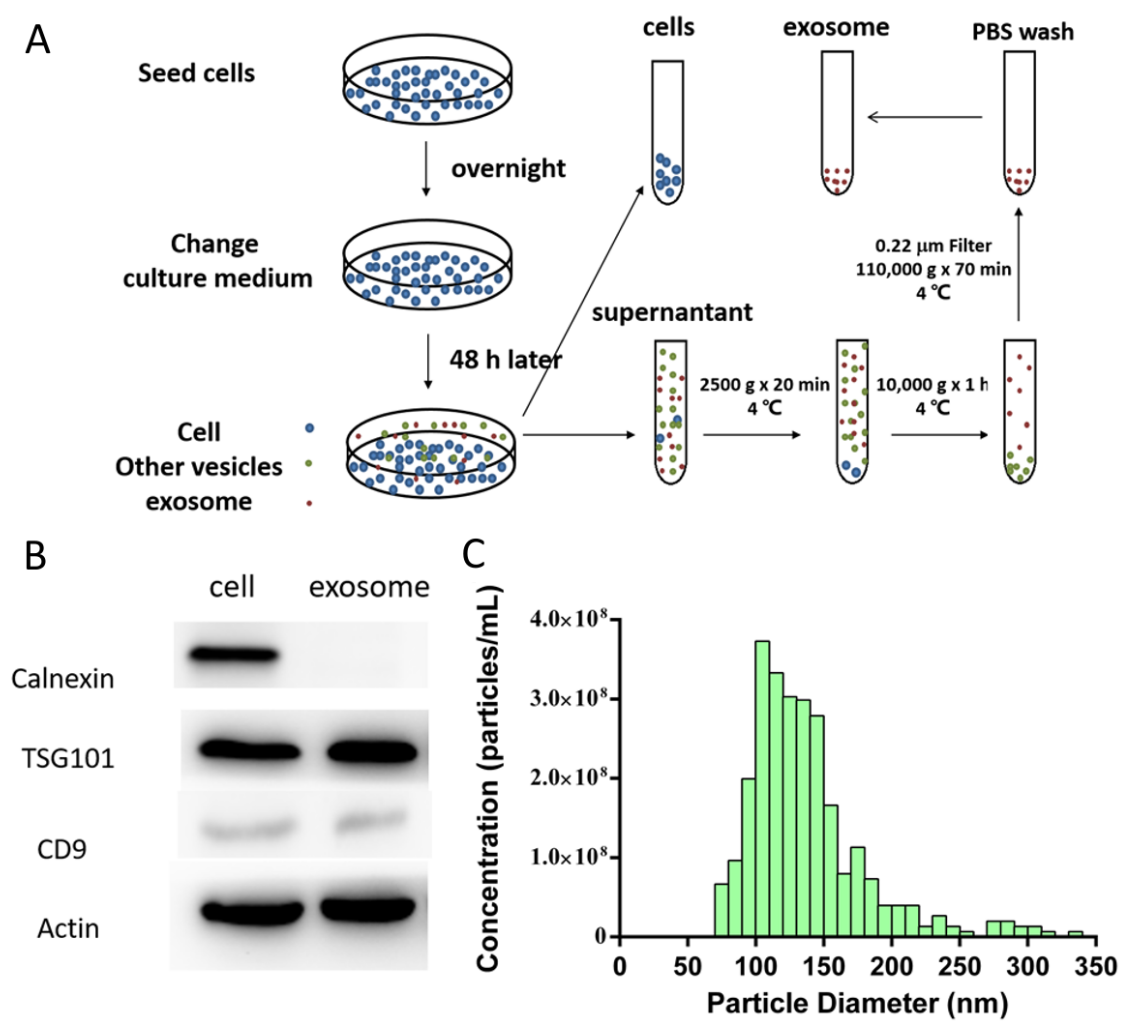

Figure 2. Isolation and characterization of exosomes. A) Protocol of exosomes isolation from cell culture medium. B) Western blot of cell and exosomes. C) The diameter distribution of exosomes.

exosomes isolated by our procedure. We also detected the diameters of exosomes and found that they generally ranged from 75 to $250 \mathrm{~nm}$, with an average mode size of $105 \pm 3.5 \mathrm{~nm}$ (Figure 2C).

Exosomal miR-19b participates in oxaliplatin sensitivity. Based on the results of Fang et al. [22], 10 miRNAs were selected, and QPCR was used to analyze the differences in levels between untreated sw480 cells and sw480 cells treated with $20 \mu \mathrm{M}$ oxaliplatin for an extended period [21]. miR-19b was the miRNA which attracted our attention (Figure 3A), because our previous studies proved that miR-19b functions as an oncogene in lung cancer and breast cancer [23], and miR-19a also functions similarly in colorectal cancer [24].

We used Targetscan, miRDB, PITA and miRanda to predict the target genes of $\mathrm{miR}-19 \mathrm{~b}$. There were 4,452 genes in miRanda, 514 genes in miRDB, 5,574 genes in PITA and 3,107 genes in Targetscan. Of these, only 437 genes were found in all four databases (Figure 3B). The DAVID database was used to analyze the enrichment for target genes [25]. Overall, 77 GO terms and 20 KEGG pathways were revealed, according to false discovery rate $(\mathrm{FDR})<0.05$. The top 10 enriched GO terms based on FDR for miR-19b targets are shown in Figure 3C. Most target genes related to protein binding, intracellular membrane-bound organelles and calcium ion binding. A count number greater than 2 and a p-value less than 0.05 were set as the cut-off values in the KEGG enrichment analysis. The top ten signal pathways are shown in Figure 3D.

miR-19b inhibits sw480 cell oxaliplatin sensitivity. To detect the role of miR-19b in oxaliplatin sensitivity, we overexpressed or knocked down miR-19b in sw480 cells. As anticipated, the miR-19b levels in sw480 cells were increased by the miR-19b mimic and decreased by the miR-19b inhibitor (Figure 4A). Compared with the control, miR-19b mimics suppressed oxaliplatin sensitivity, whereas miR-19b inhibitors promoted sensitivity (Figure 4B). In addition, we used the EdU assay to test the proliferation of cells, and it showed similar results to those in the CCK8 assay (Figure 4C).

Gw4869 suppresses the oxaliplatin sensitivity of sw480 cells by decreasing exosomal miR-19b release. Figure $5 \mathrm{~A}$ shows the chemical structure of gw 4869 . We used $10 \mu \mathrm{M}$ gw4869 to treat cells and found that it significantly inhibits the release of exosomes (Figure 5B) with a similar particle size distribution (Figure 5C). We then used gw4869 to treat sw480 cells under different concentrations of oxaliplatin and found that the IC50 of sw480 was increased (Figure 6A). The EdU assay also showed that gw4869 could suppress the oxaliplatin sensitivity of sw480 cells (Figure 6B). At the same time, we used QPCR to detect the level of miR-19b in cells and exosomes and found that gw4869 increases intracel- 
A

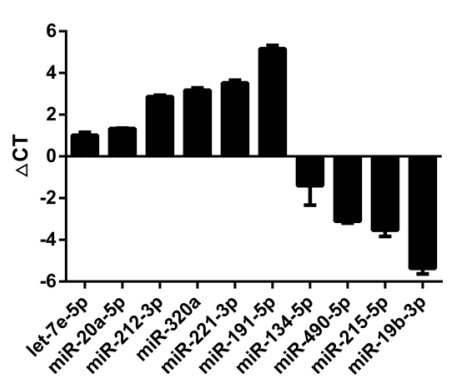

C

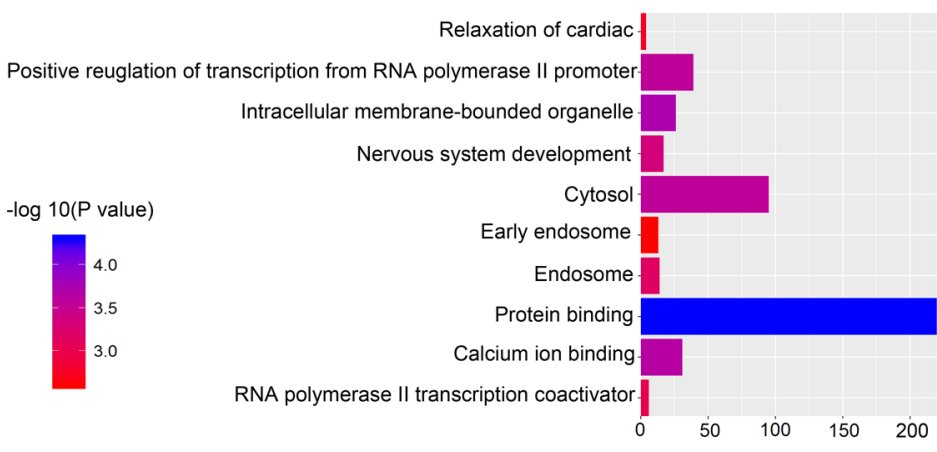

B

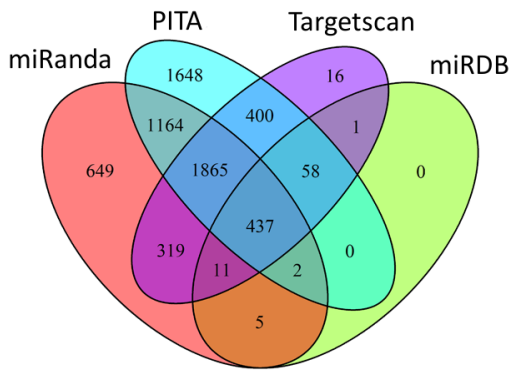

D

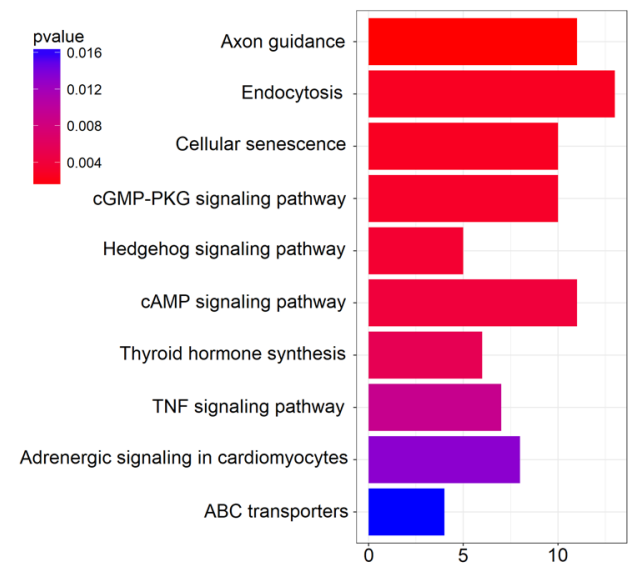

Figure 3. Profiling of exosomal miRNA expression and the bioinformatics analysis of miR-19b. A) QPCR of the level of ten miRNAs in cells. B) Prediction of miR-19b target genes. C) GO analysis. D) KEGG pathway analysis. ${ }^{\star * *}, \mathbf{p}<0.001$.

A

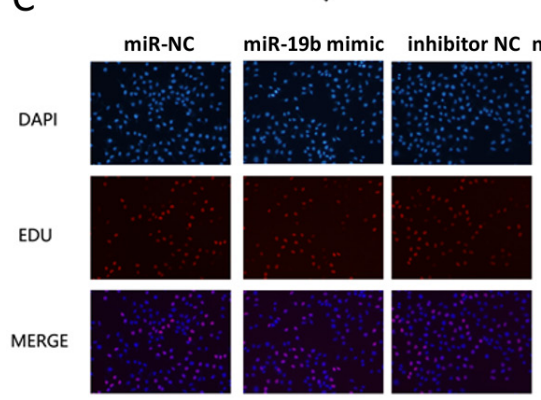

B

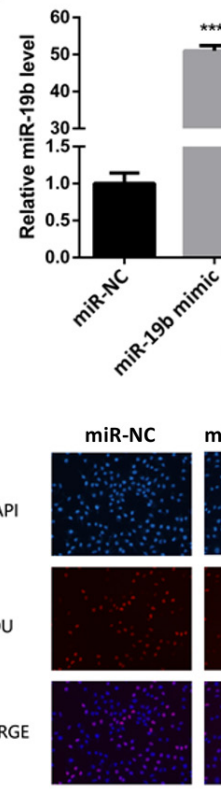

$+$

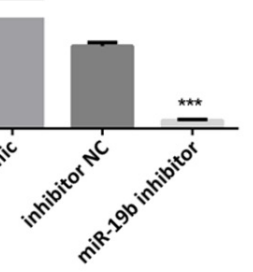

miR-19b mimic inhibitor NC miR-19b inhibitor
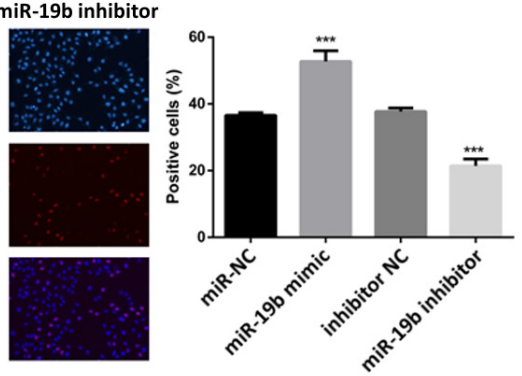

Figure 4. Function of miR-19b in regulating oxaliplatin sensitivity. A) QPCR of miR-19b level in cells transfected with miR-NC, miR-19b mimic, inhibitor NC and miR-19b inhibitor. B) The IC50 of transfected cells. C) The EdU assay of transfected cells with $20 \mu$ M oxaliplatin. Left, representative image; right: quantitative analysis. ${ }^{* *}, \mathrm{p}<0.001$. lular levels of miR-19b (Figure 6C) while decreasing the exosomal miR-19b levels (Figure 6D). To further understand the role of miR-19b and gw4869 in oxaliplatinregulation, we used gw4869 to treat sw480 cells transfected with the miR-19b mimic. We found that co-treating cells by miR-19b mimic and gw4869 had a better effect on suppressing oxaliplatin sensitivity than individual use (Suppl. Figure 1).

\section{Discussion}

Exosomes can be released from almost all living cells into the extracellular space and then influence other cells. Recent studies have shown that chemotherapy can influence the secretion of exosomes in tumors, resulting in the transfer of contents to the surrounding cells and altering their sensitivity to chemotherapy $[26,27]$. To isolate exosomes from cell culture medium, we used ultracentrifugation, an exosome isolation kit (Life technology) and ExtraPEG [28]. Considering the purity, cost and 
academic recognition of the techniques, we finally chose ultracentrifugation to isolate exosomes (data not shown). By depleting cell debris and larger vesicles, we obtained relatively pure exosomes identified by Western blot and particle size analysis.

We verified a series of differentially expressed miRNAs related to drug sensitivity by QPCR, and miR-19b showed the most obvious changes in all the differentially expressed miRNAs. Our previous studies made us particularly interested in miR-19b miRNA which is part of the miR-17-92 miRNA cluster and involved in regulating many kinds of cancers.

We have previously proven that miR-19a and miR-19b can suppress MTUS1 to promote lung cancer cell proliferation and migration [22]. Further, miR-19b promotes tumor development in breast cancer by suppressing PTPRG [23] and regulates TIA 1 and promotes cell proliferation and migration in colorectal cancer [24]. However, being oncogenic does not necessarily mean that it will promote drug resistance. Therefore, herein we showed for the first time that miR-19b suppresses sw480 cell sensitivity to oxalyplatin.

To investigate the role of miR-19b in drug sensitivity, we used bioinformatics to predict its target genes and pathways.

Of the top ten pathways of $\mathrm{GO}$ enrichment, axon guidance, adrenergic signaling in cardiomyocytes and thyroid hormone synthesis may not be directly related to drug sensitivity in cancer; and especially perhaps in colorectal cancer. Endocytosis affects the intracellular uptake of certain kinds of drugs [29] and the cGMP-PKG and cAMP signaling pathways affect cancer cell proliferation and differentiation.

Further, the TNF signaling pathway affects cell senescence and apoptosis. However, $\mathrm{ABC}$ transporters, including $\mathrm{ABCA} 1 / \mathrm{ABCB} 7 / \mathrm{ABCD} 3$ and $\mathrm{ABCC} 9$ have important roles in multi-drug resistance; with over-expression of $\mathrm{ABC}$ transporters on the plasma membrane a classic mechanism conferring cancer drug resistance [30]. The Hedgehog signaling pathway could also stimulate the survival of colorectal cancer cells [31], because its activation is essential for stem cell survival and expansion [32].

Gw4869 is a nSMase 2 (neutral sphingomyelinase 2) small molecule inhibitor which regulates ceramide levels through sphingomyelin hydrolysis. This then affects exosome release by triggering MVB inward budding [33]. We confirmed that gw4869 could suppress the secretion of exosomes in sw480 cells and then showed that gw4869 down-regulates sensitivity to oxaliplatin in these cells. While gw4869 treatment down-regulated cellular miR-19b levels the exosomal levels were up-regulated by oxaliplatin. With increased miR-19b maintained inside the cell to combat drug sensitivity, sw 480 could survive and grow under pressure from oxaliplatin.

However, the specific molecular regulation mechanism of exosomal miR-19b release remains a fascinating question. In addition, due to various restrictions, we used only miR-19b in our research, and we are sure that other exosomal miRNAs can also regulate drug sensitivity.
A

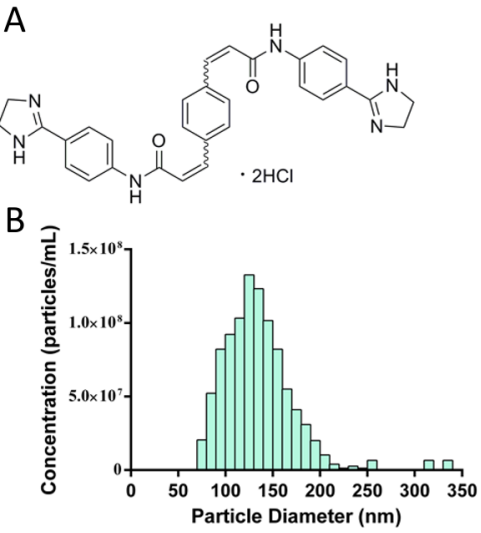

C

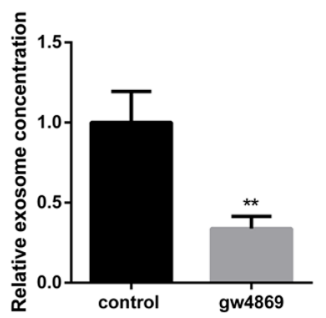

Figure 5. Gw4869 reduces exosome secretion. A) The chemical structure of gw4869. B) The diameter distribution of exosomes treated by $10 \mu \mathrm{M}$ gw4869. C) The concentration of exosomes treated by gw4869. ${ }^{* *}, \mathrm{p}<0.01$.

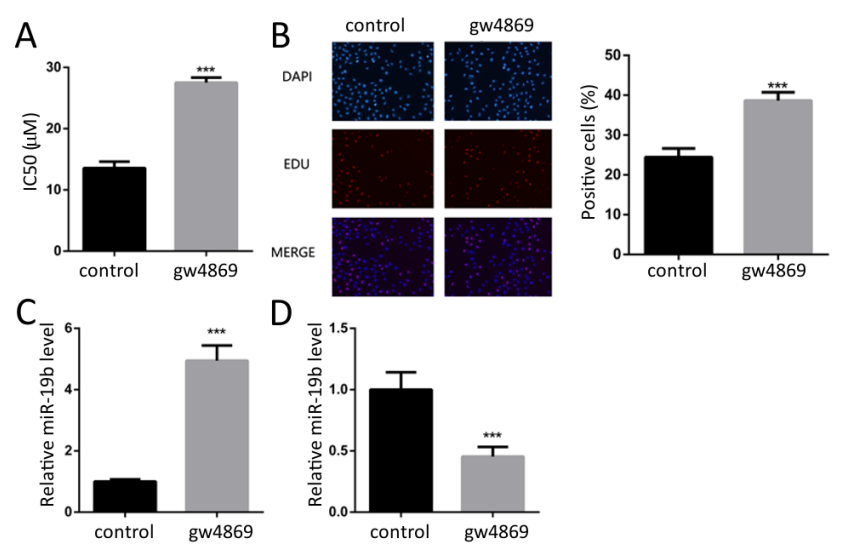

Figure 6. Gw4869 reduces oxaliplatin sensitivity of sw480 cells by suppressing exosomal miR-19b secretion. A) The IC50 of cells treated with gw4869. B) The EdU assay of cells treated with gw4869 and $20 \mu \mathrm{M}$ oxaliplatin. Left, representative image; right: quantitative analysis. C) QPCR analysis of miR-19b level in cells. D) QPCR analysis of miR-19b level in exosomes. ${ }^{\star * *}, \mathrm{p}<0.001$

Finally, our combined results indicate for that first time, that gw4869 regulates sw 480 cell oxaliplatin sensitivity by suppressing exosomal miR-19b release. This method could provide a novel mechanism for overcoming the drug resistance encountered in colorectal cancer.

Supplementary information is available in the online version of the paper.

References

[1] BAREFORD LM1, SWAAN PW. Endocytic mechanisms for targeted drug delivery. Adv Drug Deliv Rev 2007; 8: 748758. https://doi.org/10.1016/j.addr.2007.06.008 
[2] BARTEL DP. MicroRNAs: genomics, biogenesis, mechanism, and function. Cell 2004; 2: 281-297. https://doi.org/10.1016/ S0092-8674(04)00045-5

[3] BERMAN DM, KARHADKAR SS, MAITRA A, MONTES DE OCA R, GERSTENBLITH MR et al. Widespread requirement for Hedgehog ligand stimulation in growth of digestive tract tumors. Nature 2003; 6960: 846-851. https:// doi.org/10.1038/nature01972

[4] DEEN KI, SILVA H, DEEN R, CHANDRASINGHE PC. Colorectal cancer in the young, many questions, few answers. World J Gastrointest Oncol 2016; 6: 481-488. https:// doi.org/10.4251/wjgo.v8.i6.481

[5] ESSANDOH K, YANG L, WANG X, HUANG W, QIN D et al. Blockade of exosome generation with GW4869 dampens the sepsis-induced inflammation and cardiac dysfunction. Biochim Biophys Acta 2015;1852: 2362-2371. https://doi. org/10.1016/j.bbadis.2015.08.010

[6] FANG L, LI H, WANG L, HU J, JIN T et al. MicroRNA-17-5p promotes chemotherapeutic drug resistance and tumor metastasis of colorectal cancer by repressing PTEN expression. Oncotarget 2014; 10: 2974-2987. https://doi.org/10.18632/ oncotarget.1614

[7] FILIPOWICZ W, BHATTACHARYYA SN, SONENBERG $\mathrm{N}$. Mechanisms of post-transcriptional regulation by microRNAs: are the answers in sight? Nat Rev Genet 2008; 2: 102-114. https://doi.org/10.1038/nrg2290

[8] GOLDBERG RM, SARGENT DJ, MORTON RF, FUCHS CS, RAMANATHAN RK et al. A randomized controlled trial of fluorouracil plus leucovorin, irinotecan, and oxaliplatin combinations in patients with previously untreated metastatic colorectal cancer. J Clin Oncol 2004; 1: 23-30. https:// doi.org/10.1200/JCO.2004.09.046

[9] GRAHAM J, MUSHIN M, KIRKPATRICK P. Oxaliplatin. Nat Rev Drug Discov 2004; 1: 11-12. https://doi.org/10.1038/ nrd1287

[10] GU Y, LIU S, ZHANG X, CHEN G, LIANG H et al. Oncogenic miR-19a and miR-19b co-regulate tumor suppressor MTUS1 to promote cell proliferation and migration in lung cancer. Protein Cell 2017; 6: 455-466. https://doi. org/10.1007/s13238-017-0393-7

[11] HANNAFON BN, DING WQ. Intercellular communication by exosome-derived microRNAs in cancer. Int J Mol Sci 2013; 7: 14240-14269. https://doi.org/10.3390/ijms140714240

[12] HU Y, YAN C, MU L, HUANG K, LI X et al. FibroblastDerived Exosomes Contribute to Chemoresistance through Priming Cancer Stem Cells in Colorectal Cancer. PLoS One 2015; 5: e125625. https://doi.org/10.1371/journal. pone. 0125625

[13] HUANG DA W, SHERMAN BT, LEMPICKI RA. Systematic and integrative analysis of large gene lists using DAVID bioinformatics resources. Nat Protoc 2009; 1: 44-57. https://doi. org/10.1038/nprot.2008.211

[14] JI R, ZHANG B, ZHANG X, XUE J, YUAN X et al. Exosomes derived from human mesenchymal stem cells confer drug resistance in gastric cancer. Cell Cycle 2015; 15: 24732483. https://doi.org/10.1080/15384101.2015.1005530
[15] KHARAZIHA P, CEDER S, LI Q, PANARETAKIS T. Tumor cell-derived exosomes: a message in a bottle. Biochim Biophys Acta 2012; 1826: 103-111. https://doi.org/10.1016/j. bbcan.2012.03.006

[16] LI J, LIU K, LIU Y, XU Y, ZHANG F et al. Exosomes mediate the cell-to-cell transmission of IFN-alpha-induced antiviral activity. Nat Immunol 2013; 8: 793-803. https://doi. org/10.1038/ni.2647

[17] LIU M, YANG R, URREHMAN U, YE C, YAN X et al. MiR$19 \mathrm{~b}$ suppresses PTPRG to promote breast tumorigenesis. Oncotarget 2016; 39: 64100-64108. https://doi.org/10.18632/ oncotarget.11799

[18] LIU Y, GU Y, HAN Y, ZHANG Q, JIANG Z et al. Tumor Exosomal RNAs Promote Lung Pre-metastatic Niche Formation by Activating Alveolar Epithelial TLR3 to Recruit Neutrophils. Cancer Cell 2016; 30: 243-256. https://doi. org/10.1016/j.ccell.2016.06.021

[19 LIU Y, LIU R, YANG F, CHENG R, CHEN X et al. miR19a promotes colorectal cancer proliferation and migration by targeting TIA1. Mol Cancer 2017; 16: 53. https://doi. org/10.1186/s12943-017-0625-8

[20] MULLER L, MITSUHASHI M, SIMMS P GOODING WE5, WHITESIDE TL. Tumor-derived exosomes regulate expression of immune function-related genes in human $\mathrm{T}$ cell subsets. Sci Rep 2016; 20254. https://doi.org/10.1038/ srep20254

[21] NOBILI S, GALLETTA A, BRUGIA M, TASSI R, PETRENI $P$ et al. Emerging drugs in refractory colorectal cancer. Future Med Chem 2015; 12: 1491-1501. https://doi.org/10.4155/ fmc. 15.85

[22] RABENECK L, HORTON S, ZAUBER AG, EARLE C. Colorectal Cancer. pp 101-119. In: H. Gelband, P. Jha, R. Sankaranarayanan, S. Horton (Eds). Cancer: Disease Control Priorities, Third Edition. World Bank, Washington (DC) 2015, p 363. ISBN 978-1-4648-0369-7. https://doi. org/10.1596/978-1-4648-0349-9

[23] RAFTERY L, SANOFF HK, GOLDBERG R. Colon cancer in older adults. Semin Oncol 2008; 6: 561-568. https://doi. org/10.1053/j.seminoncol.2008.08.004

[24] RIDER MA, HURWITZ SN, MECKES DG JR. ExtraPEG: A Polyethylene Glycol-Based Method for Enrichment of Extracellular Vesicles. Sci Rep 2016; 23978. https://doi. org/10.1038/srep23978

[25] SHELKE GV, LASSER C, GHO YS, LOTVALL J. Importance of exosome depletion protocols to eliminate functional and RNA-containing extracellular vesicles from fetal bovine serum. J Extracell Vesicles 2014; 3. https://doi.org/10.3402/ jev.v3.24783

[26] SYN N, WANG L, SETHI G, THIERY JP, GOH BC. Exosome-Mediated Metastasis: From Epithelial-Mesenchymal Transition to Escape from Immunosurveillance. Trends Pharmacol Sci 2016; 37: 606-617. https://doi.org/10.1016/j. tips.2016.04.006

[27] SZAKACS G, PATERSON JK, LUDWIG JA, BOOTHGENTHE C, GOTTESMAN MM. Targeting multidrug resistance in cancer. Nat Rev Drug Discov 2006; 3: 219-234. https://doi.org/10.1038/nrd1984 
[28] TKACH M, THERY C. Communication by Extracellular Vesicles: Where We Are and Where We Need to Go. Cell 2016; 164: 1226-1232. https://doi.org/10.1016/j.cell.2016.01.043

[29] TRAJKOVIC K, HSU C, CHIANTIA S, RAJENDRAN L, WENZEL $\mathrm{D}$ et al. Ceramide triggers budding of exosome vesicles into multivesicular endosomes. Science 2008; 5867: 1244-1247. https://doi.org/10.1126/science.1153124

[30] VALADI H, EKSTROM K, BOSSIOS A, SJOSTRAND M, LEE JJ et al. Exosome-mediated transfer of mRNAs and microRNAs is a novel mechanism of genetic exchange between cells. Nat Cell Biol 2007; 6: 654-659. https://doi.org/10.1038/ ncb1596

[31] VARNAT F, DUQUET A, MALERBA M, ZBINDEN M, MAS C et al. Human colon cancer epithelial cells harbour active HEDGEHOG-GLI signaling that is essential for tumor growth, recurrence, metastasis and stem cell survival and expansion. EMBO Mol Med 2009; 6-7: 338-351. https:// doi.org/10.1002/emmm.200900039
[32] WANG X, HUANG W, LIU G, CAI W, MILLARD RW et al. Cardiomyocytes mediate anti-angiogenesis in type 2 diabetic rats through the exosomal transfer of miR-320 into endothelial cells. J Mol Cell Cardiol 2014; 74: 139-150. https://doi. org/10.1016/j.yjmcc.2014.05.001

[33] ZHAI Z, YU X, YANG B, ZHANG Y, ZHANG L et al. Colorectal Cancer Heterogeneity and Targeted Therapy: Clinical Implications, Challenges and Solutions for Treatment Resistance. Semin Cell Dev Biol 2017; 64: 107-115. https://doi.org/10.1016/j.semcdb.2016.08.03 


\section{Suppressing the secretion of exosomal miR-19b by gw4869 could regulate oxaliplatin sensitivity in colorectal cancer}

Y. Y. GU, J. YU, J. F. ZHANG*, C. WANG*

Supplemental Material

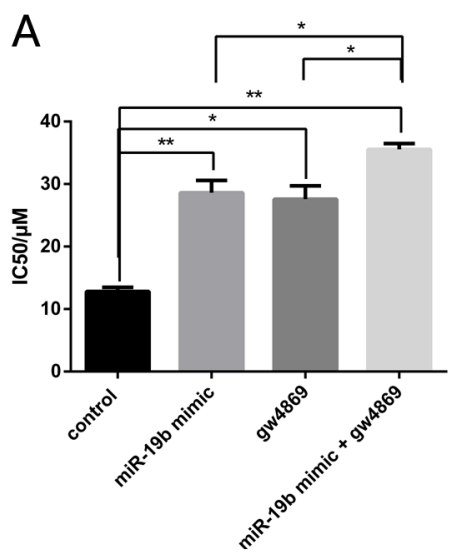

B

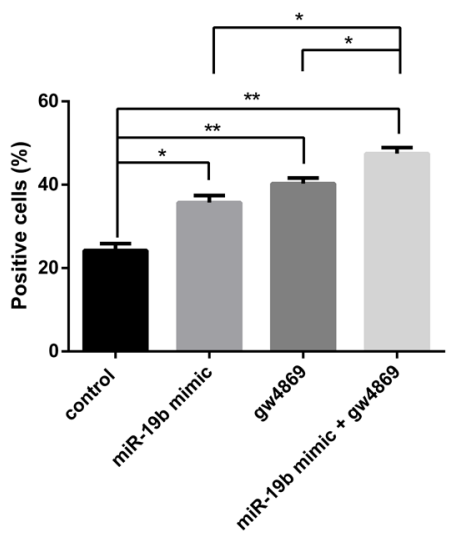

Supplementary Figure 1. The effect of co-treatment by miR-19b mimic and gw4869. A) The IC50 of cells treated with gw4869. B) The EdU assay of cells treated with gw 4869 and $20 \mu \mathrm{M}$ oxaliplatin. Left, representative image; right: quantitative analysis. ${ }^{\star}, \mathrm{p}<0.05$. $^{* *}, \mathrm{p}<0.01$. 